\title{
Phylogenetic Diversity of Rhizoctonia solani Associated with Canola and Wheat in Alberta, Manitoba, and Saskatchewan
}

K. D. Broders, Department of Biological Sciences, University of New Hampshire, Durham NH 03824; M. L. Parker, M. S. Melzer, and G. J. Boland, School of Environmental Sciences, University of Guelph, Guelph ON, Canada N1G 2W1

\begin{abstract}
Broders, K. D., Parker, M. L., Melzer, M. S., and Boland, G. J. 2014. Phylogenetic diversity of Rhizoctonia solani associated with canola and wheat in Alberta, Manitoba, and Saskatchewan. Plant Dis. 98:1695-1701.

Rhizoctonia solani is a damaging soilborne pathogen, which affects most field crops in the Canadian provinces of Alberta, Manitoba, and Saskatchewan. The objective of this study was to conduct a phylogenetic comparison of isolates of $R$. solani collected from a previous survey in the major canola- and wheat-growing regions of western Canada. A total of 128 multinucleate isolates from a previous survey were identified by internal transcribed spacer (ITS) sequence and compared to anastomosis group (AG) results. The multinucleate isolates of $R$. solani were grouped into eight distinct clades. Each clade corresponded to a specific AG with the exception of two distinct clades that

were observed for isolates classified as AG 2-1 by anastomosis testing. While most isolates of AG 5 clustered together according to ITS sequences, three isolates classified by anastomosis grouping as AG 5 grouped with AG 2-1, AG 4, and a binucleate Rhizoctonia sp. in the phylogenetic analysis. In most instances, the results from AG tests were consistent with ITS sequence, but there were still several cases where isolates were inconsistently classified or failed to undergo anastomosis with any of the tester strains used in this study. This provides support for the use of the ITS region as a valuable tool for rapid identification of $R$. solani isolates to their respective AGs.
\end{abstract}

Globally, Rhizoctonia solani is an important soilborne pathogen of a number of agriculturally important crops causing damping-off, foliar blight, and root and stem rot. The species is highly genetically diverse and has been separated into independent anastomosis groups (AGs) based on the ability of strains to successfully fuse and exchange nuclear material with known tester strains. Several recent studies have attempted to use molecular approaches to separate AGs or lineages within $R$. solani. The genetic relatedness of $R$. solani AGs was evaluated by Kuninaga et al. (14), and they found that a percent similarity higher than $96 \%$ of the internal transcribed spacer (ITS) sequence was evident for isolates within an AG subgroup, 66 to $100 \%$ for isolates of different subgroups within an AG, and 55 to $96 \%$ for isolates among different AGs. AG 2 was found to be especially diverse and includes a number of subgroups, which are subdivided according to their morphology, host range, virulence, and hyphal anastomosis frequency. Currently, subgroups 2-1, 2-2 IIIB, 2-2 IV, 2-2 LP, 2-2 WB, 2-3, 2-4, and 2-BI are included in AG $2(2,6)$, and ITS sequencing analysis was consistent with the above listed subgroups $(2,26,27)$. In addition, the rDNAITS sequences accurately divided subgroups within AG 1 (14), AG 3 (14), AG $4(1,14)$, and AG 6 (21).

The wide host range of $R$. solani extends from dicotyledonous plants, including canola, soybeans, dry beans and peas, to monocotyledonous plants, such as wheat and corn. The majority of reports of $R$. solani on cereals originate from Australia (16) and the northwest region of the United States (18), where AG 8 is the most commonly detected subgroup. In a survey of wheat in Texas, AG 4

\section{Corresponding author: K. D. Broders, E-mail: kirk.broders@unh.edu}

Current address of M. L. Parker: International Potato Center (CIP), Regional Office Sub-Saharan Africa, ILRI Campus, P.O. Box 25171-00603, Nairobi, Kenya.

Current address of M. S. Melzer: Pest Diagnostic Clinic, Laboratory Services Division, University of Guelph, Guelph, ON, Canada, N1H 8J7.

Accepted for publication 1 June 2014.

http://dx.doi.org/10.1094/PDIS-02-14-0146-RE

(C) 2014 The American Phytopathological Society was most frequently recovered in addition to several isolates of $A G$ 2-2 and AG 5 (25). $R$. solani is also becoming an increasingly important pathogen on canola as production of this crop continues to expand beyond Canada and North Dakota into the northwestern United States and Australia, and extends into new areas such as South Africa. Most reports have found that AG 2-1 is the most frequently recovered $R$. solani AG from canola $(9,12,15,20,37,38)$. However, several recent studies have isolated other AGs from canola, including AG 10 (28) and Ceratobasidium spp. in Washington (29), and AG 3, AG 4-HGII, AGA, AG I, and AG K in South Africa (35). Even though wheat and canola are the two major crops in western Canada, little is known about the diversity of Rhizoctonia spp. infecting them.

A recent survey by Melzer et al. (17) evaluated the diversity of Rhizoctonia species from barley, bean, canola, pea, soybean, and wheat crops in Canada. They found that $R$. solani isolated from canola and wheat included members of AG 2-1, 4, and 5 (17). However, 17 isolates were not successfully assigned an AG group. This was likely due to the fact that only four tester strains (AG 2-1, 2-2, 4, and 5) were available for anastomosis testing of all isolates, and the unassigned strains may belong to different AG groups than those four tested. Therefore, if isolates from AG groups other than those four, or from novel AG groups, were recovered, they would go unidentified. The alternative strategy currently being used for classification of $R$. solani isolates has relied on differences in the sequence of the ITS regions of their rDNA. Analysis of the rDNAITS region was shown to be a reliable method to identify Rhizoctonia spp. $(32,33)$. In a recent survey of Rhizoctonia in Europe, Goll et al. (7) identified 288 isolates of Rhizoctonia by ITS sequences, and the determination of $\mathrm{AG}$ was based on the homology of the sequence from the unknown isolate to that of an appropriate set of reference sequences from the NCBI database. As the ITS region can vary substantially even among isolates of an AG, the authors set the positive identification level at $85 \%$ and found that, in $27 \%$ of the cases, homology was $<95 \%$. It is that $27 \%$ that may represent distinct groups within an AG, but without a proper phylogenetic analysis it is difficult to determine which AGs are highly conserved and which have a high level of variability both within and among AGs.

Given the potential shortfalls of both identification methods mentioned above, the aims of this study were to: (i) conduct a phylogenetic analysis of isolates of $R$. solani collected from canola 
and wheat from western Canada; and (ii) determine if classical anastomosis grouping using tester strains is consistent with results from phylogenetic analyses.

\section{Materials and Methods}

Rhizoctonia isolates and determination of anastomosis groups. Rhizoctonia isolates used in this study were obtained through the survey of Melzer et al. (17) as a subset of isolates from western Canada. Details of these survey methods and results are presented elsewhere (17). In brief, during May, June, and July of 2009 to 2011, canola, pea, and wheat crops were surveyed in Alberta, Saskatchewan, and Manitoba. The majority of crops were sampled at the seedling stage, but some crops were sampled at later stages of plant development. When available, the cropping history of sampled fields was recorded. A diamond-shaped pattern of $50 \mathrm{~m}$ per side was used for sampling. Twenty seedlings were collected per field, with one seedling sampled every $10 \mathrm{~m}$ around each sides of the diamond. If areas with symptoms of disease were observed in the crop during sampling, additional seedlings were collected from these diseased areas. Seedlings were stored at $4{ }^{\circ} \mathrm{C}$ until being processed. Seedlings were rinsed in cool water, upper stems and leaves were removed, and remaining crowns and roots were then surface-disinfested for $30 \mathrm{~s}$ in $70 \%$ ethanol followed by $90 \mathrm{~s}$ in $0.6 \%$ sodium hypochlorite. Seedling crowns and roots were rinsed, blotted dry, then plated on water agar (WA). Isolates of Rhizoctonia spp. were identified using hyphal and colony characteristics, and transferred to acidified potato dextrose agar (Difco; PDA) medium (APDA). Isolates were stored on APDA slants at $7^{\circ} \mathrm{C}$.

Table 1. Isolates of Rhizoctonia solani collected from canola, wheat, and pea in Alberta, Manitoba, and Saskatchewan, Canada ${ }^{\mathrm{a}}$

\begin{tabular}{|c|c|c|c|c|}
\hline Isolate & Province & Host & AG group ${ }^{b}$ & Accession number \\
\hline 10-C-F_5C & Alberta & Canola & AG 2-1 & KF870886 \\
\hline 10-C-F_16C & Alberta & Canola & AG 2-1 & KF870887 \\
\hline 10-C-F_20C & Alberta & Canola & AG 2-1 & KF870888 \\
\hline 10-C-T_7R & Alberta & Canola & AG 2-1 & KF870889 \\
\hline 10-C-T_13C & Alberta & Canola & AG 2-1 & KF870890 \\
\hline 10-D4_16R & Alberta & Pea & AG 2-1 & KF870891 \\
\hline 10-D5_3C & Alberta & Wheat & AG 2-1 & KF870892 \\
\hline 10-D5_12C & Alberta & Wheat & AG unk & KF870893 \\
\hline 11-A2_16R & Alberta & Wheat & AG 2-1 & KF870894 \\
\hline 11-A4_10C & Alberta & Canola & AG 2-1 & KF870895 \\
\hline 11-A7_4C & Alberta & Wheat & AG 5 & KF870896 \\
\hline 11-A10_9C & Alberta & Wheat & AG 5 & KF870897 \\
\hline 11-A10_10C & Alberta & Wheat & AG 5 & KF870898 \\
\hline 11-A10_11C & Alberta & Wheat & AG 5 & KF870899 \\
\hline 11-A10_16C & Alberta & Wheat & AG 5 & KF870900 \\
\hline D4_5R & Alberta & Canola & AG 2-1 & KF870901 \\
\hline D4_9C & Alberta & Canola & AG 2-1 & KF870902 \\
\hline D4_12_C & Alberta & Canola & AG 2-1 & KF870903 \\
\hline D4_14R & Alberta & Canola & AG 2-1 & KF870904 \\
\hline D5_12C & Alberta & Canola & AG 2-1 & KF870905 \\
\hline D5_13C & Alberta & Canola & AG 2-1 & KF870906 \\
\hline D5_19_C & Alberta & Canola & AG unk & KF870907 \\
\hline D5_19S & Alberta & Canola & AG 2-1 & KF870908 \\
\hline D6_16C & Alberta & Canola & AG 2-1 & KF870909 \\
\hline M1_1C & Alberta & Wheat & AG 4 & KF870910 \\
\hline M1_2S & Alberta & Wheat & AG 4 & KF870911 \\
\hline M1_6S & Alberta & Wheat & AG 4 & KF870912 \\
\hline M1_8S & Alberta & Wheat & AG 4 & KF870913 \\
\hline M1_9C & Alberta & Wheat & $\mathrm{AG} 4$ & KF870914 \\
\hline M1_12C & Alberta & Wheat & AG 4 & KF870915 \\
\hline 10-C-5_10R & Manitoba & Canola & AG 2-1 & KF870916 \\
\hline 10-C-5_19C & Manitoba & Canola & AG 2-1 & KF870917 \\
\hline 10-D-10_9C & Manitoba & Wheat & AG 2-1 & KF870918 \\
\hline 10-D-10_11C & Manitoba & Wheat & AG 5 & KF870919 \\
\hline 10-M13_8C & Manitoba & Canola & AG 2-1 & KF870920 \\
\hline 10-M13_19C & Manitoba & Canola & AG 2-1 & KF870921 \\
\hline 10-M13S_5C & Manitoba & Canola & AG 2-1 & KF870922 \\
\hline 10-M13S_6C & Manitoba & Canola & AG 2-1 & KF870923 \\
\hline 11-M11_14C & Manitoba & Canola & AG 2-1 & KF870924 \\
\hline 11-M11_20C & Manitoba & Canola & AG 2-1 & KF870925 \\
\hline M6_14C & Manitoba & Wheat & AG 2-1 & KF870926 \\
\hline M7_2C & Manitoba & Wheat & AG 2-1 & KF870927 \\
\hline M7_3C & Manitoba & Wheat & AG 4 & KF870928 \\
\hline M7_4C & Manitoba & Wheat & AG 2-1 & KF870929 \\
\hline M7_7C & Manitoba & Wheat & AG 2-1 & KF870930 \\
\hline M7_8C & Manitoba & Wheat & AG 2-1 & KF870931 \\
\hline M7_10R & Manitoba & Wheat & AG 2-1 & KF870932 \\
\hline M7_11R & Manitoba & Wheat & AG 2-1 & KF870933 \\
\hline M7_12C & Manitoba & Wheat & AG 4 & KF870934 \\
\hline M7_16R & Manitoba & Wheat & AG 4 & KF870935 \\
\hline M9_16R & Manitoba & Wheat & AG unk & KF870936 \\
\hline M9_18C & Manitoba & Wheat & AG 5 & KF870937 \\
\hline M9_19R & Manitoba & Wheat & AG 2-1 & KF870938 \\
\hline
\end{tabular}

${ }^{a}$ Isolates were assigned to an AG group based on successful anastomosis with known tester strains AG 2-1, AG 2-2, AG 4, and AG 5.

${ }^{\mathrm{b}}$ Indicates the isolate was able to successfully anastomose with a known tester strain of the AG. Those isolates that did not anastomose with tester strains used in this study are labeled AG unk (unknown). 
Hyphae of isolates of Rhizoctonia spp. were stained with trypan blue $(0.5 \%$ in lactophenol) and examined microscopically for the number of nuclei per cell to determine if the isolates were binucleate or multinucleate. Multinucleate isolates were considered to be $R$. solani. To determine the anastomosis groups of the recovered multinucleate $R$. solani isolates, an agar plug was taken from the actively growing margin of an isolate of unknown AG and plated on WA 2 to $3 \mathrm{~cm}$ apart from an isolate of known AG. Isolates were considered to be in the same AG when anastomosis was observed a minimum of five times.

DNA extraction, polymerase chain reaction (PCR) amplification and sequencing. DNA was extracted from mycelia cultured on PDA lined with cellophane (Flexel Sales Inc.), using the Power
Plant Pro DNA Isolation Kit (MoBio Laboratories Inc.). Samples were stored at $-20^{\circ} \mathrm{C}$. PCR was performed in $50-\mu l$ reaction volume containing $10 \mu \mathrm{l}$ of $5 \times$ Green GoTaq reaction buffer (Promega Corp.), $5 \mu \mathrm{l}$ of $25 \mathrm{mM} \mathrm{MgCl} 2,1 \mu$ containing $10 \mathrm{mM}$ each dNTP, $0.25 \mu \mathrm{l}$ of GoTaq Taq DNA polymerase, $5 \mu \mathrm{l}$ of each primer ITS4 and ITS5 (36) at $5 \mathrm{pmol}^{-1} \mathrm{l}^{-1}, 2 \mu \mathrm{l}$ of DNA, and $21.75 \mu \mathrm{l}$ of sterile distilled water. The PCR amplification conditions were initial denaturation at $95^{\circ} \mathrm{C}$ for $2 \mathrm{~min}$, followed by 40 cycles of $15 \mathrm{~s}$ at $95^{\circ} \mathrm{C}, 1 \mathrm{~min}$ at $57^{\circ} \mathrm{C}$, and $30 \mathrm{~s}$ at $72^{\circ} \mathrm{C}$, with a final $10 \mathrm{~min}$ at $72^{\circ} \mathrm{C}$. Amplified products were purified using Qiaquick spin columns (Qiagen Inc.). DNA was quantified using a NanoDrop 1000 spectrophotometer (Thermo Scientific). Amplified products were sequenced by including $14 \mathrm{ng}$ of purified DNA with the BigDye

Table 1. (continued from previous page)

\begin{tabular}{|c|c|c|c|c|}
\hline Isolate & Province & Host & AG group ${ }^{\mathbf{b}}$ & Accession number \\
\hline M10_1C & Manitoba & Wheat & AG 2-1 & KF870939 \\
\hline M10_2C & Manitoba & Wheat & AG 5 & KF870940 \\
\hline M10_3C & Manitoba & Wheat & AG 4 & KF870941 \\
\hline M10_9C & Manitoba & Wheat & AG 4 & KF870942 \\
\hline M10_13C & Manitoba & Wheat & AG 4 & KF870943 \\
\hline M10_18C & Manitoba & Wheat & AG 4 & KF870944 \\
\hline M10_21C & Manitoba & Wheat & AG 4 & KF870945 \\
\hline M10_23C & Manitoba & Wheat & AG 5 & KF870946 \\
\hline M11_1R & Manitoba & Wheat & AG 2-1 & KF870947 \\
\hline M11_4R & Manitoba & Wheat & AG 5 & KF870948 \\
\hline M11_9R & Manitoba & Wheat & AG 2-1 & KF870949 \\
\hline M11_16C & Manitoba & Wheat & AG 5 & KF870950 \\
\hline M11_18C & Manitoba & Wheat & AG 5 & KF870951 \\
\hline M11_24R & Manitoba & Wheat & AG 5 & KF870952 \\
\hline 10-C-P1_4C & Saskatchewan & Canola & AG 2-1 & KF870953 \\
\hline 10-C-P1_5C & Saskatchewan & Canola & AG unk & KF870954 \\
\hline 10-C-P1_6C & Saskatchewan & Canola & AG 2-1 & KF870955 \\
\hline 10-C-P1_7R & Saskatchewan & Canola & AG 2-1 & KF870956 \\
\hline 10-C-P1_8C & Saskatchewan & Canola & AG 2-1 & KF870957 \\
\hline 10-C-P1_11C & Saskatchewan & Canola & AG 2-1 & KF870958 \\
\hline 10-C-P1_12C & Saskatchewan & Canola & AG 2-1 & KF870959 \\
\hline 10-C-P1_13C & Saskatchewan & Canola & AG 2-1 & KF870960 \\
\hline 10-C-P1_15C & Saskatchewan & Canola & AG 2-1 & KF870961 \\
\hline 10-C-P1_16C & Saskatchewan & Canola & AG 2-1 & KF870962 \\
\hline 10-C-P1_17R & Saskatchewan & Canola & AG 2-1 & KF870963 \\
\hline 10-C-P1_18C & Saskatchewan & Canola & AG 2-1 & KF870964 \\
\hline 10-C-P1_20C & Saskatchewan & Canola & AG 2-1 & KF870965 \\
\hline 10-C-P3_10C & Saskatchewan & Canola & AG 2-1 & KF870966 \\
\hline 10-C-P3_15C & Saskatchewan & Canola & AG 2-1 & KF870967 \\
\hline 10-D7-13_12_C & Saskatchewan & Canola & AG 2-1 & KF870968 \\
\hline $11-\mathrm{S} 3 \_4 \mathrm{C}$ & Saskatchewan & Canola & AG 2-1 & KF870969 \\
\hline 11-S3_10C & Saskatchewan & Canola & AG 2-1 & KF870970 \\
\hline 11-S3_14C & Saskatchewan & Canola & AG 2-1 & KF870971 \\
\hline 11-S4_14C & Saskatchewan & Canola & AG 2-1 & KF870972 \\
\hline 11-S4_15C & Saskatchewan & Canola & AG 2-1 & KF870973 \\
\hline 11-S4_16C & Saskatchewan & Canola & AG 2-1 & KF870974 \\
\hline 11-S4_20C & Saskatchewan & Canola & AG 2-1 & KF870975 \\
\hline 11-S5_1C & Saskatchewan & Wheat & AG 2-1 & KF870976 \\
\hline 11-S5_2C & Saskatchewan & Wheat & AG 2-1 & KF870977 \\
\hline 11-S5_4C & Saskatchewan & Wheat & AG 2-1 & KF870978 \\
\hline 11-S5_6C & Saskatchewan & Wheat & AG 2-1 & KF870979 \\
\hline 11-S5_7C & Saskatchewan & Wheat & AG 2-1 & KF870980 \\
\hline 11-S5_8C & Saskatchewan & Wheat & AG 2-1 & KF870981 \\
\hline 11-S5_9C & Saskatchewan & Wheat & AG unk & KF870982 \\
\hline 11-S5_10C & Saskatchewan & Wheat & AG 2-1 & KF870983 \\
\hline 11-S5_11C & Saskatchewan & Wheat & AG 2-1 & KF870984 \\
\hline 11-S5_13R & Saskatchewan & Wheat & AG 2-1 & KF870985 \\
\hline 11-S5_14C & Saskatchewan & Wheat & AG 2-1 & KF870986 \\
\hline 11-S5_15C & Saskatchewan & Wheat & AG 2-1 & KF870987 \\
\hline 11-S6_6C & Saskatchewan & Canola & AG 2-1 & KF870988 \\
\hline 11-S6_7C & Saskatchewan & Canola & AG 2-1 & KF870989 \\
\hline 11-S6_11C & Saskatchewan & Canola & AG 2-1 & KF870990 \\
\hline 11-S6_15C & Saskatchewan & Canola & AG 2-1 & KF870991 \\
\hline 11-S6_16C & Saskatchewan & Canola & AG 2-1 & KF870992 \\
\hline 11-S6_18C & Saskatchewan & Canola & AG 2-1 & KF870993 \\
\hline 11-S6_19C & Saskatchewan & Canola & AG 2-1 & KF870994 \\
\hline CMC2_9C & Saskatchewan & Canola & AG unk & KF870995 \\
\hline D7_11C & Saskatchewan & Canola & AG 2-1 & KF870996 \\
\hline D8__13R & Saskatchewan & Canola & AG 2-1 & KF870997 \\
\hline
\end{tabular}




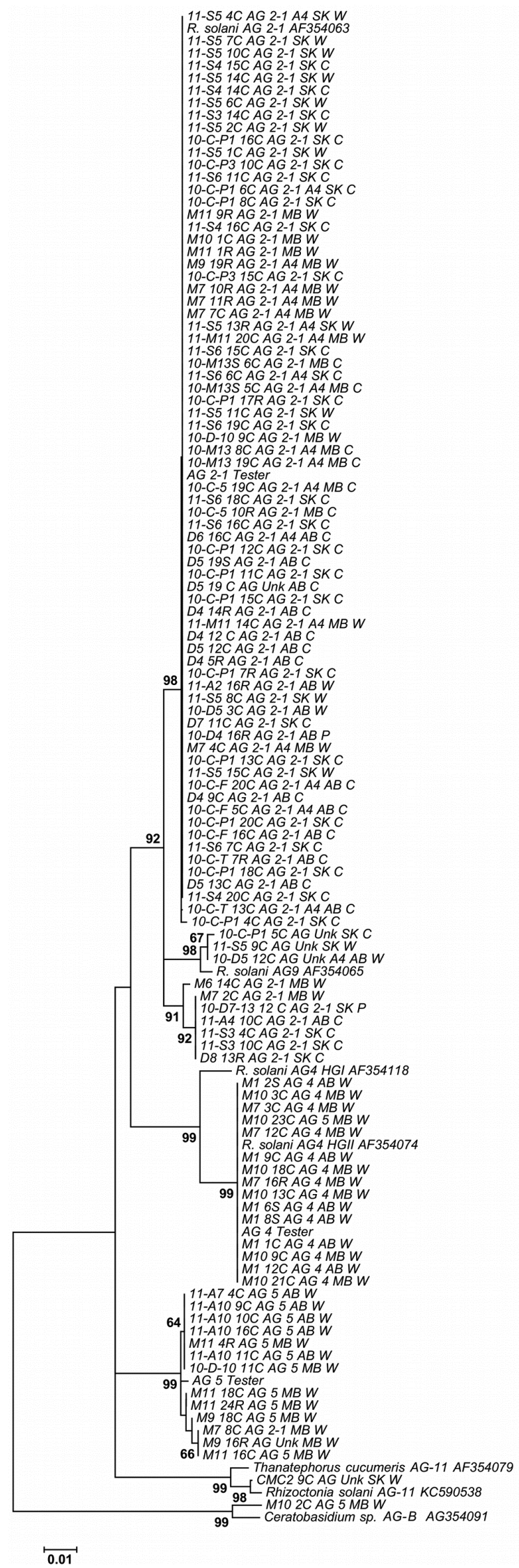

Fig. 1. Maximum likelihood tree of the internal transcribed spacer (ITS) sequence of 112 isolates of Rhizoctonia recovered from canola (c), pea (p), and wheat (w) from Alberta (AB), Manitoba (MB), and Saskatchewan (SK). Isolate name consists of isolate number, $A G$ group from anastomosis test, province (AB, MB, or $S K)$, and crop (C, P, or W). Isolates imported from GenBank are followed by an accession number. Posterior probabilities $>60 \%$ are displayed at the nodes. version 3.1 Ready Reaction Kit (Applied BioSystems) on an ABI 3730 DNA Analyzer (Applied BioSystems) at the University of Guelph Genomics Facility. Forward and reverse sequences were assembled and aligned using Geneious Pro software (v.5.4.3). All sequences were deposited in the NCBI GenBank, and accession numbers were included in Table 1.

Phylogenetic analysis. To infer species, AGs, and outgroups, reference sequences from studies by Sharon et al. $(31,33)$, Gonzalez et al. (8), or from vouchered cultures in the Centraalbureau voor Schimmelcultures (CBS) fungal biodiversity center were imported from GenBank. The phylogenetic and molecular analyses were completed using MEGA version 4.0 (13). Alignments were completed using ClustalW, and trees were inferred using the neighbor joining (NJ) method (Kimura two-parameter distance calculation). Gaps were treated as missing data with missing or ambiguous sites ignored for the affected pairwise comparison. All positions were included in the analyses, and relative support for the branches was estimated with 1,000 bootstrap replications (5) for $\mathrm{NJ}$ analysis. Analysis of polymorphic sites was completed using DnaSP (24).

Phylogenetic trees were also inferred using Bayesian inference as implemented in MrBayes v. 3.1.2 (10). A Bayesian analysis using the general time reversible (GTR) model was selected for the entire unpartitioned alignment, with likelihood parameter settings (lset) number of substitution types (nst) $=6$, with a proportion of sites invariable and the rest drawn from the gamma distribution (rate = invgamma). Four independent analyses, each starting from a random tree, were run under the same conditions for the combined gene alignment. Three hot and one cold chain Markov Chain Monte Carlo with 1,000,000 generations with sampling every 100 generations was used for the analysis. The first 250,000 generations were discarded as the chains were converging (burn-in period).

Diversity within AGs was also assessed by collapsing sequences down into haplotypes using SNAP workbench 2.0 (22), to enable analysis of the clone-corrected data set. In the case of isolates belonging to AG 5, a set of $95 \%$ plausible haplotype networks connecting the haplotypes by mutational steps was constructed using statistical parsimony in the program TCS (3). Due to the genetic distance between isolates belonging to AG 2-1 and AG-9, rather than completing a haplotype network, a phylogenetic analysis similar to the one described above was completed. In addition to the analysis of the AG 2-1 haplotypes, isolates listed as AG 2-1 in NCBI GenBank were imported to evaluate the similarity of AG 2-1 isolated from canola and wheat in Canada to other isolates of AG 2-1 from around the world.

\section{Results}

For the majority of multinucleate isolates of $R$. solani recovered from Alberta, Manitoba, and Saskatchewan, phylogenetic identification against known sequences from GenBank corresponded consistently to the results of AG tester strains. While anastomosis testing grouped the isolates into four AG groups, analysis of the ITS sequences grouped the isolates into six distinct clades corresponding with AG 2-1, AG 4, AG 5, AG 9, AG 11, and Ceratobasidium sp. AG B (Fig. 1), and seven isolates that formed hyphal fusions with the AG 2-1 tester strain but formed a distinct seventh clade that did not have a close sequence match to any of the known AG groups in GenBank (Fig. 1). The separation of these two groups was supported with strong posterior probability support (92\%) and may represent two distinct lineages that retain the ability to exchange genetic material but perhaps only rarely. AG 2-1 was the most frequently recovered anastomosis group in all three provinces surveyed in this study by both anastomosis testing and ITS sequences.

There were three instances where isolates successfully fused with an AG tester strain but were found to be more closely related to another AG group based on ITS sequence data. In one instance, an isolate (M7 8C) successfully fused with an AG 2-1 tester strain, but sequence data indicated it was a member of AG 5. The second 
and third instances were isolates M10 23C and M10 2C, respectively, that successfully fused with AG 5 , but sequences indicated these isolates were more closely related to AG 4 and Ceratobasidium sp. AG B, respectively. There were six isolates that did not successfully fuse with the tester strains AG 2-1, AG 2-2, AG 4, and AG 5. In two of these instances, the isolate belonged to an AG group based on ITS sequence data but did not fuse with the corresponding tester strain. One of the isolates clustered with members of AG 2-1 (D5 19C), another isolate (M9 16R) clustered with members of AG 5. After the identity of these isolates was determined, they were re-evaluated with their respective tester strains to ensure that their inability to anastomose was not an error. The results from the repeated tests proved that neither isolate was able to successfully fuse with the corresponding tester strain. Of the remaining four isolates that did not fuse with tester strains used in this study, three clustered with strong bootstrap support to an isolate of AG 9 used by Gonzalez et al. (8), and one isolate was found to be most similar to an isolate of AG 11 also used in the study by Gonzalez et al. (8). Tester strains of these two AG groups were not available to evaluate the ability of unknowns to anastomose with members of their respective groups.

No genetic diversity was observed among isolates classified as AG 4, as all 15 isolates formed a single haplotype. In contrast, multiple haplotypes of AG 2-1, AG 5, and AG 9 were observed. In the case of AG 5, the 13 isolates were separated into seven haplotypes with seven isolates grouping together in haplotype H5, and the remaining haplotypes were represented by a single isolate each (Fig. 2). The haplotype map provides a similar result to the phylogenetic tree, which has the seven isolates of $\mathrm{H} 5$ separated from the other members of AG 5 with strong bootstrap support (97\%). In the case of AG 2-1, the 83 isolates were grouped into 15 haplotypes. In the phylogenetic analysis, these 15 haplotypes grouped into three distinct clades with strong posterior probability (80\%) (Fig. 3). The BLAST search found that several isolates of AG 2-1 have been previously recovered from canola in Alberta. In addition, the isolates of AG 2-1 from the prairies of Canada have close similarity to isolates recovered from potato in France and Finland, cabbage in New York, ginseng in China, cauliflower in Belgium, and Brassica oleracea in the United Kingdom, demonstrating the global distribution and host range of this important soilborne pathogen. Finally, the three isolates of AG 9 were grouped into two haplotypes and had the greatest similarity to isolates of AG 9 from $B$. oleracea from the United Kingdom.

Wheat plants hosted the greatest diversity of $R$. solani AGs including strains from AG 2-1, AG 4, AG 5, AG 9, AG 11, and an unclassified AG (Fig. 4). All isolates of AG 4 and AG 5 from this study were recovered from wheat. In contrast, all isolates recovered from canola belonged to AG 2-1, with four exceptions. A single isolate grouped with AG 9, while three other isolates clustered with the unclassified AG group. There was no geographical clustering of any of the AG groups across the three provinces. Representatives from six clades were found in Alberta and Manitoba (Fig. 5). No isolates from AG 4 or AG 5 clades were recovered in Saskatchewan, but the only isolate of AG 11 was found there (Fig. 5).

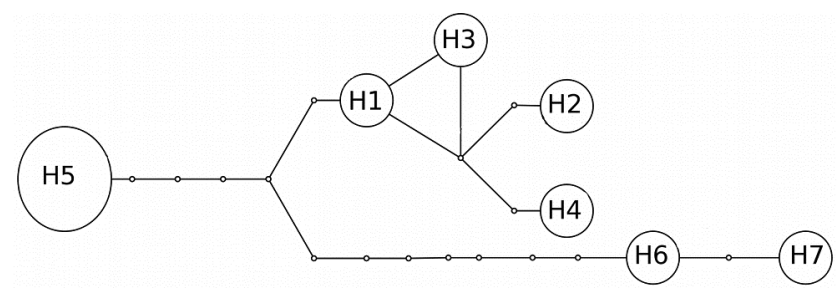

Fig. 2. Most parsimonious haplotype network for 7 haplotypes of Rhizoctonia solani AG-5 recovered from wheat in Alberta and Manitoba, Canada, derived from the internal transcribed spacer (ITS) gene region. Sizes of haplotype circles indicate frequencies of the haplotypes. Each line represents a single nucleotide mutation, and small empty circles represent unsampled haplotypes.

\section{Discussion}

Molecular characterization of $R$. solani in the provinces of Alberta, Manitoba, and Saskatchewan showed the presence of a diverse assemblage of AG groups including AG 2-1, AG 4, AG 5, AG 9, AG 11, and one isolate of Ceratobasidium (Fig. 1). The dominant group was AG 2-1, and this was likely due to the fact that nearly half of the isolates used in this study were recovered from canola. The high frequency of AG 2-1 associated with canola is consistent with previous reports from this crop $(11,12,15,39)$. In contrast to canola, wheat harbored a diverse group of isolates belonging to six AG groups and one isolate of Ceratobasidium (Fig. 2). Of these groups, AG 2-1, AG 4, and AG 5 were the most frequently recovered. Both AG 4 and AG 5 were shown to consistently cause disease on wheat (17). However, while AG 2-1 was consistently isolated from wheat roots, the isolates of this AG do not typically cause disease on wheat (17). Wheat appears to be harboring isolates of AG 2-1, allowing inoculum levels to remain high enough to cause disease on successive canola crops. This represents the first documentation of the diversity of $R$. solani recovered from wheat in Canada, which is unexpected given the importance of the crop to central Canada and previous reports of $R$. solani on canola in the same region. Interestingly, $R$. solani AG 8 was not recovered in our study area, which is the most common AG on wheat grown in the in Pacific Northwest of the United States (18) and in Australia (16). However, in a more recent survey of Rhizoctonia spp. from wheat soils across 14 states in the United States, AG 2-1 was the most frequently recovered strain in addition to AG 3, AG 4, AG 10, and AG 11 (30). These results are consistent with this study with the exception that AG 3 and AG 10 were not found in our study sites, and AG 5 and AG 9 were not recovered in the United States. However, AG 5 as well as AG 2-1 and AG 4

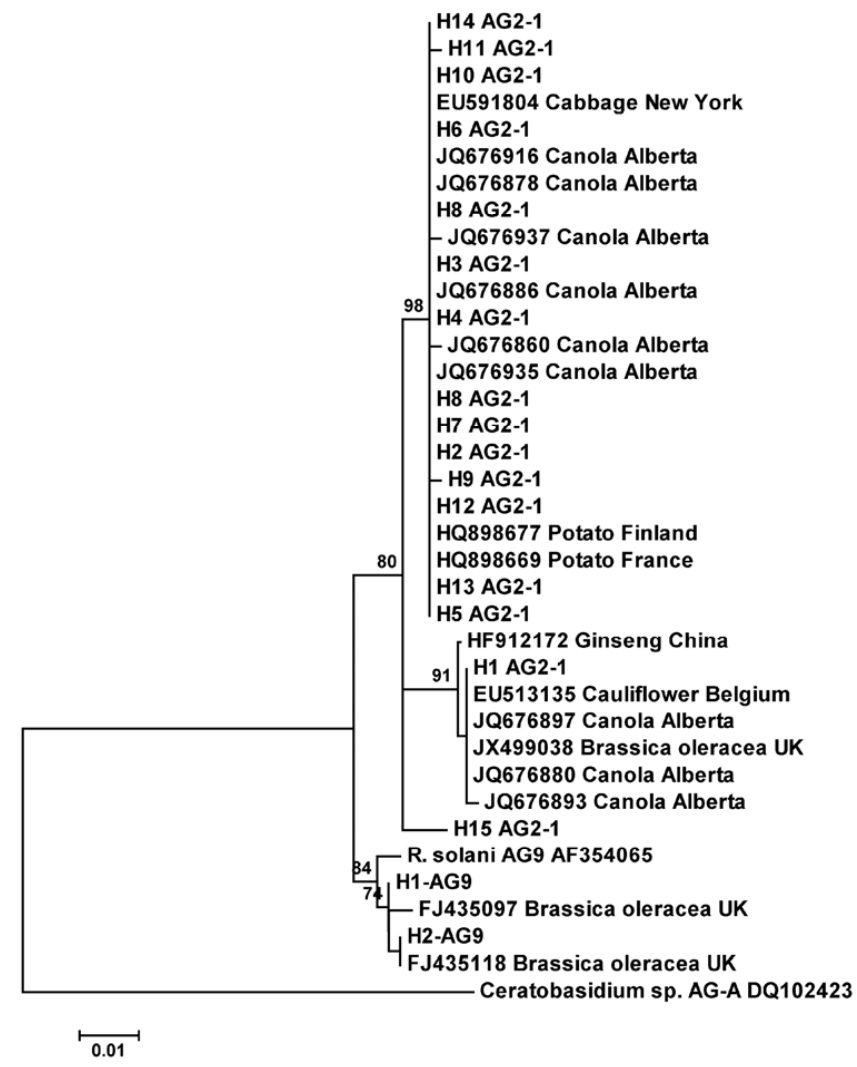

Fig. 3. Maximum likelihood tree of the internal transcribed spacer (ITS) sequence of 15 haplotypes of the Rhizoctonia solani AG2-1 complex and 2 haplotypes AG-9, recovered from canola, pea, and wheat from Alberta, Manitoba, and Saskatchewan. Isolates imported from GenBank include accession number, crop, and location the isolate was recovered from. Posterior probabilities $>70 \%$ are displayed at the nodes. 
were reported from wheat in Turkey (4). In a recent survey conducted across several European countries, Rhizoctonia species were baited from agricultural soils and AG 9 and AG 4 were the most abundant AGs found, but a number of other AGs were also reported, including AG 1, AG 2-1, AG 3, AG 5, AG 8, and AG 11 (7). However, as these isolates were baited from the soil, it only provides information on which AGs are most abundant and not which AGs are pathogenic on specific crops that may be planted in that soil. While this is the only other recent survey to report the presence of AG 9 in wheat-producing areas, none of the isolates were recovered from wheat plants, and therefore it is difficult to determine the importance of this AG as a potential pathogen of wheat.

In the study by Melzer et al. (17), isolates of $R$. solani recovered from western Canada were tested for pathogenicity on canola, lentil, pea, soybean, and wheat in growth room studies. All of the

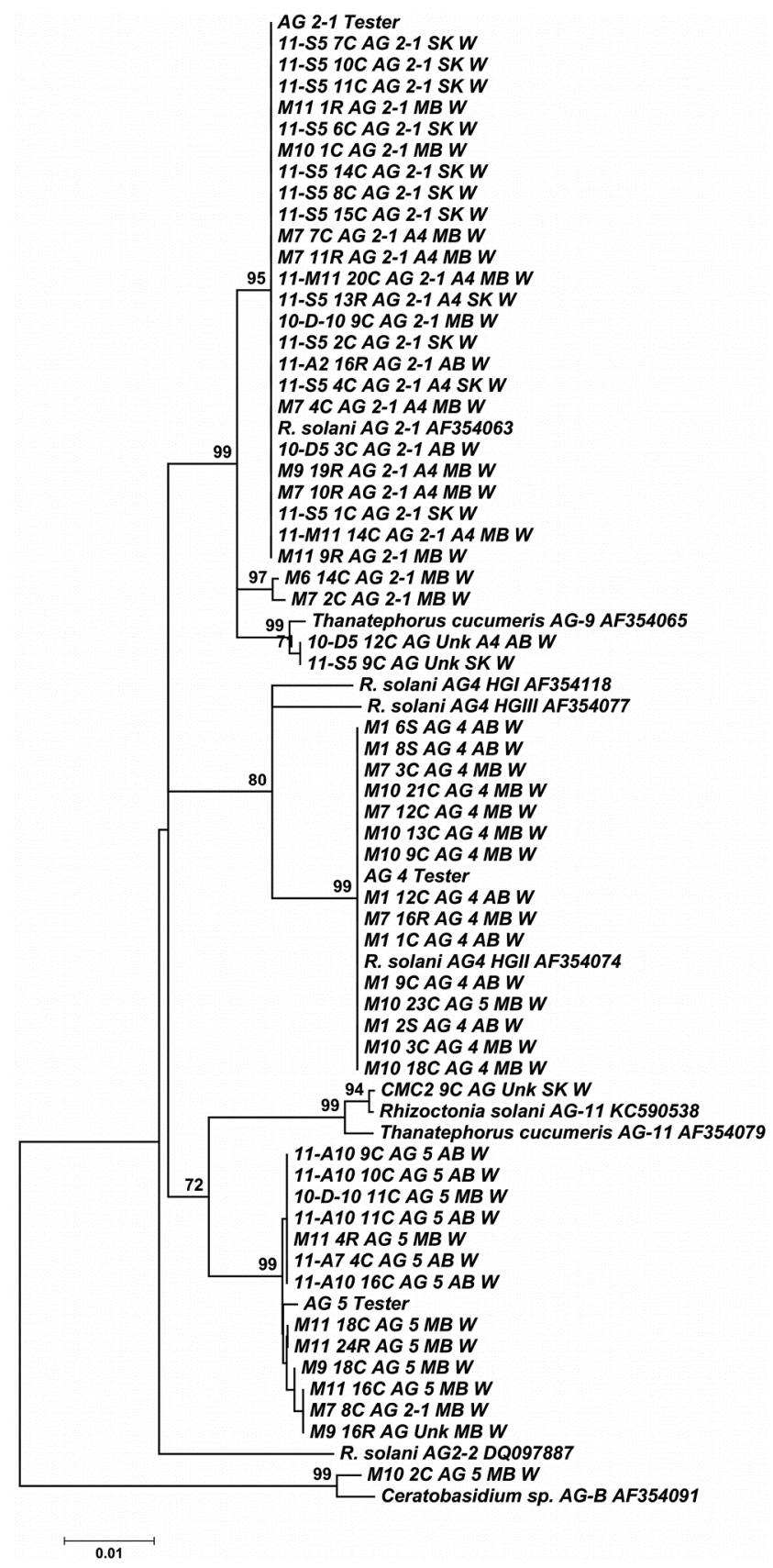

Fig. 4. Maximum likelihood tree from the analysis of the internal transcribed spacer (ITS) sequences of 55 isolates of Rhizoctonia solani recovered from wheat (w) from Alberta (AB), Manitoba (MB), and Saskatchewan (SK). Isolate name consists of isolate number, $A G$ group from anastomosis test, province (AB, MB, or SK), and crop (W). Posterior probability $>70 \%$ included at nodes. isolates in AG 2-1 were pathogenic on canola, but only 37, 21, and $5 \%$ of the 94 isolates tested were pathogenic on lentil, pea, and wheat, respectively. There were eight isolates identified as AG 2-1 that were pathogenic on canola, lentil, and pea, and seven of these isolates clustered together to form the second AG 2-1 clade (Fig. 1), providing further support for a distinct subgroup in AG 2-1 that may be based on host range. Isolates of unknown AG in the clade with known isolates of AG 9 were not pathogenic on hosts tested or had low virulence (17). Isolates of AG 4 were pathogenic on all hosts tested, and isolates of AG 5 were pathogenic on wheat, lentil, and canola. AG 5 was not as virulent on these hosts as isolates from $\mathrm{AG} 4$.

While AG tests were consistent with ITS sequence most of the time, approximately $10 \%$ of isolates were incorrectly classified or failed to undergo anastomosis with tester strains used in this study, and the ITS gene proved effective at rapidly classifying these isolates. This provides further evidence of the utility of the ITS gene in identifying a greater number of isolates, including rare AGs, in large surveys. This also supports several other previous studies that have demonstrated that grouping of Rhizoctonia isolates by molecular analysis based on the ITS sequences supports the AG grouping of Rhizoctonia isolates based on classical hyphal anastomosis reactions $(19,23,33,34)$.

While ITS remains the preferred method for rapid assignment of $R$. solani isolates to their respective AGs, there remains a number of drawbacks to using this region. As $R$. solani is multinucleate, there is the potential for a strain to possess multiple ITS sequences that vary by one or more nucleotides $(26,27)$. This generally results in hard-to-interpret chromatograms. The authors have observed this complication in the case of isolates of $R$. solani recovered from soybean and dry bean that belong to AG 2-2. Preliminary data indicate that many strains have at least four independent ITS sequences. This study also provides evidence that there are likely a number of distinct lineages or species that make up the $R$. solani complex, with strong posterior probability for separation of lineages. However, further study with a greater number of nuclear and mitochondrial genes is needed.

Finally, a previously undocumented AG group was identified, and the AG 5 tester strain was found to form hyphal fusions with several unrelated groups of multinucleate and binucleate $R h i$ zoctonia spp., proving there are still a number of questions regarding species boundaries and potential for genetic exchange through hyphal fusion in $R$. solani.

\section{Acknowledgments}

This investigation is based upon work supported, in part, by the National Sciences and Engineering Research Council of Canada (NSERC) and Syngenta Canada Inc.

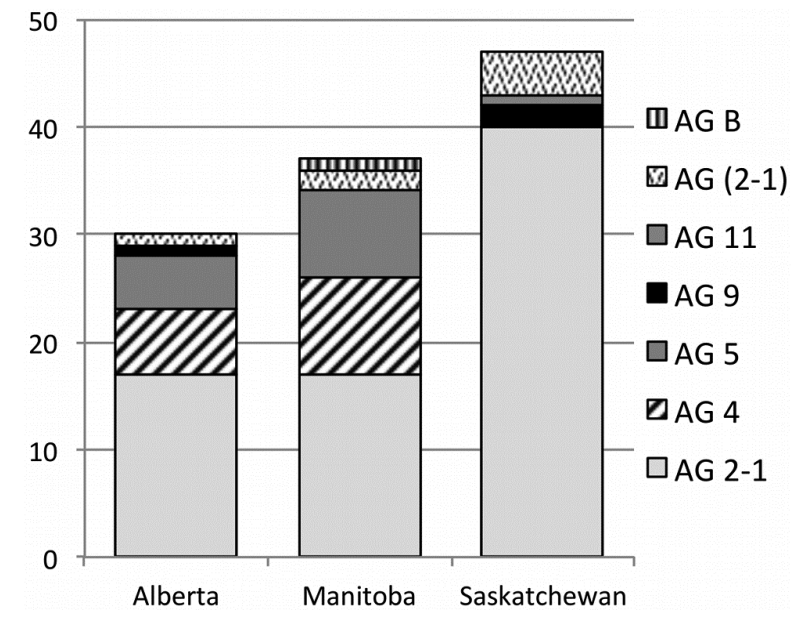

Fig. 5. Distribution of Rhizoctonia solani isolates belonging to seven anastomosis groups present in Alberta, Manitoba, and Saskatchewan, Canada. Isolates identified as AG (2-1) represent those strains which successfully anastomosed with tester strain AG 2-1, but formed a distinct group in the phylogenetic analysis. 


\section{Literature Cited}

1. Boysen, M., Borja, M., delMoral, C., Salazar, O., and Rubio, V. 1996. Identification at strain level of Rhizoctonia solani AG4 isolates by direct sequence of asymmetric PCR products of the ITS regions. Curr. Genet. 29:174-181.

2. Carling, D. E., Kuninaga, S., and Brainard, K. A. 2002. Hyphal anastomosis reactions, rDNA-internal transcribed spacer sequences, and virulence levels among subsets of Rhizoctonia solani anastomosis group-2 (AG-2) and AGBI. Phytopathology 92:43-50.

3. Clement, M., Posada, D., and Crandall, K. A. 2000. TCS: A computer program to estimate gene genealogies. Mol. Ecol. 9:1657-2659.

4. Demirci, E. 1998. Rhizoctonia species and anastomosis groups isolated from barley and wheat in Erzum, Turkey. Plant Pathol. 47:10-15.

5. Felsenstein, J. 1985. Confidence limits on phylogenies: An approach using the bootstrap. Evolution 6:227-242.

6. Godoy-Lutz, G., Kuninaga, S., Steadman, J. R., and Powers, K. 2008. Phylogenetic analysis of Rhizoctonia solani subgroups associated with web blight symptoms on common bean based on ITS-5.8S rDNA. J. Gen. Plant Pathol. 74:32-40.

7. Goll, M. B., Schade-Schuetze, A., Swart, G., Oostendorp, M., Schott, J. J., Jaser, B., and Felsenstein, F. G. 2014. Survey of the prevalence of Rhizoctonia spp. in European soils and determination of the baseline sensitivity towards sedaxane. Plant Pathol. 63:148-154.

8. Gonzalez, D., Carling, D. E., Kuninaga, S., Vilgalys, R., and Cubeta, M. A. 2001. Ribosomal DNA systematics of Ceratobasidium and Thanatephorus with Rhizoctonia anamorphs. Mycologia 93:1138-1150.

9. Gugel, R. K., Yitbarek, S. M., Verma, P. R., Morrall, R. A. A., and Sadasivaiah, R. S. 1987. Etiology of the Rhizoctonia root rot complex of canola in the peace river region of Alberta. Can. J. Plant Pathol. 9:119-128.

10. Huelsenbeck, J. P., and Ronquist, F. 2001. MRBAYES 3: Bayesian phylogenetic inference under mixed models. Bioinformatics 19:1572-1574.

11. Hwang, S. F., Swanson, T. A., and Evans, I. R. 1986. Characterization of Rhizoctonia solani isolates from canola in west central Alberta. Plant Dis. 70:681-683.

12. Khangura, R. K., Barbetti, M. J., and Sweetingham, M. W. 1999. Characterization and pathogenicity of Rhizoctonia species on canola. Plant Dis. 83:714-721.

13. Kumar, S., Tamura, K., and Nei, M. 2004. MEGA3: Integrated software for molecular evolutionary analysis and sequence alignment. Briefings Bioinform. 5:150-163.

14. Kuninaga, S., Natsuaki, T., Takeuchi, T., and Yokosawa, R. 1997. Sequence variation of the rDNA ITS regions within and between anastomosis groups in Rhizoctonia solani. Curr. Genet. 32:237-243.

15. Lamprecht, S. C., Tewoldemedhin, Y. T., Hardy, M., Calitz, F. J., and Mazzola, M. 2011. Effect of cropping system on composition of the Rhizoctonia populations recovered from canola and lupin in a winter rainfall region of South Africa. Eur. J. Plant Pathol. 131:305-316.

16. MacNish, G. C., and Neate, S. M. 1996. Rhizoctonia bare patch of cereals An Australian perspective. Plant Dis. 80:965-971.

17. Melzer, M. S., Dickson, A., Labun, T., and Boland, G. J. Characterization and pathogenicity of Rhizoctonia spp. from field crops in Canada. Can. J. Plant Pathol. In press.

18. Ogoshi, A., Cook, R. J., and Bassett, E. N. 1990. Rhizoctonia species and anastomosis groups causing root rot of wheat and barley in the Pacific Northwest. Phytopathology 80:784-788.

19. Ohkura, M., Abawi, G. S., Smart, C. D., and Hodge, K. T. 2009. Diversity and aggressiveness of Rhizoctonia solani and Rhizoctonia-like fungi on vegetables in New York. Plant Dis. 93:615-624.

20. Paulitz, T. C., Okubara, P. A., and Schillinger, W. F. 2006. First report of damping-off of canola caused by Rhizoctonia solani AG 2-1 in Washington State. Plant Dis. 90:829.
21. Pope, E. J., and Carter, D. A. 2001. Phylogenetic placement and host specificity of mycorrhizal isolates belonging to AG-6 and AG-12 in the Rhizoctonia solani species complex. Mycologia 93:712-719.

22. Price, E. W., and Carbone, I. 2005. SNAP: Workbench management tool for evolutionary population genetic analysis. Bioinformatics 21:402-404.

23. Rinehart, T. A., Copes, W. E., Toda, T., and Cubeta, M. A. 2007. Genetic characterization of binucleate Rhizoctonia species causing web blight on azalea in Mississippi and Alabama. Plant Dis. 91:616-623.

24. Rozas, J., Sanchez-DelBarrio, J. C., Messenguer, X., and Rozas, R. 2003. DnaSP, DNA polymorphism analyses by the coalescent and other methods. Bioinformatics 19:2496-2497

25. Rush, C. M., Carling, D. E., Harverson, R. M., and Mathieson, J. T. 1994. Prevalence and pathogenicity of anastomosis groups of Rhizoctonia solani from wheat and sugarbeet in Texas. Plant Dis. 78:349-352.

26. Salazar, O., Julian, M. C., Hyakumachi, M., and Rubio, V. 2000. Phylogenetic grouping of cultural types of Rhizoctonia solani AG 2-2 based on ribosomal ITS sequences. Mycologia 92:505-509.

27. Salazar, O., Schneider, J. H. M., Julian, M. C., Keijer, J., and Rubio, V. 1999. Phylogenetic subgrouping of Rhizoctonia solani AG 2 isolates based on ribosomal ITS sequences. Mycologia 91:459-467.

28. Schroeder, K. L., and Paulitz, T. C. 2012. First report of root rot caused by Rhizoctonia solani AG-10 on canola in Washington state. Plant Dis. 96:584

29. Schroeder, K. L., and Paulitz, T. C. 2012. First report of a Ceratobasidium sp. causing root rot on canola in Washington state. Plant Dis. 96:591.

30. Schroeder, K. L., Shetty, K. K., and Paulitz, T. C. 2011. Survey of Rhizoctonia spp. from wheat soils in the US and determination of pathogenicity on wheat and barley. (Abstr.) Phytopathology 101:S161.

31. Sharon, M., Kuninaga, S., Hyakumachi, M., and Sneh, B. 2006. The advancing identification and classification of Rhizoctonia spp. using molecular and biotechnological methods compared with the classical anastomosis grouping. Mycoscience 47:299-316.

32. Sharon, M., Freeman, S., Kuninaga, S., and Sneh, B. 2007. Genetic diversity, anastomosis groups and virulence of Rhizoctonia spp. from strawberry. Eur. J. Plant Pathol. 117:247-265.

33. Sharon, M., Kuninaga, S., Hyakumachi, M., Naito, S., and Sneh, B. 2008 Classification of Rhizoctonia spp. using rDNA-ITS sequence analysis supports the genetic basis of the classical anastomosis grouping. Mycoscience 49:93-114.

34. Stodart, B. J., Harvey, P. R., Neate, S. M., Melanson, D. L., and Scott, E. S 2007. Genetic variation and pathogenicity of anastomosis group 2 isolates of Rhizoctonia solani in Australia. Mycol. Res. 111:891-900.

35. Tewoldemedhin, Y. T., Lamprecht, S. C., McLeod, A., and Mazzola, M. 2006. Characterization of Rhizoctonia spp. recovered from crop plants used in rotational cropping systems in the Western Cape Province of South Africa. Plant Dis. 90:1399-1406.

36. White, T. J., Bruns, T., Lee, S., and Taylor, J. 1990. Amplification and direct sequencing of fungal ribosomal RNA genes for phylogenetics. Pages 315322 in: PCR Protocols: A Guide to Methods and Applications. M. A. Innis, D. H. Gelfand, J. J. Sninsky, and T. J. White, eds. Academic Press, San Diego, CA.

37. Yang, J., and Verma, P. R. 1992. Screening genotypes for resistance to preemergence damping off and post emergence seedling root rot of oilseed rape and canola caused by Rhizoctonia solani AG2-1. Crop Prot. 11:443448.

38. Yitbarek, S. M., Verma, P. R., and Morrall, R. A. A. 1987. Anastomosis groups, pathogenicity, and specificity of Rhizoctonia solani isolates from seedling and adult rapeseed canola plant and soils in Saskatchewan. Can. J. Plant Pathol. 9:6-13.

39. Zhou, Q.-X., Chang, K.-F., Hwang, S.-F., Strelkov, S. E., Gossen, B. D., and Chen, Y.-Y. 2009. Pathogenicity and genetic diversity of Rhizoctonia solan isolates from lupin and other crops in Alberta, Canada. Can. J. Plant Pathol. 31:340-347. 